\title{
DOE/SF/19460..TI
}

\section{Diagnosis of High-Temperature Implosions \\ Using Low- and High-Opacity Krypton Lines}

\author{
B. YAAKOBI and R. EPSTEIN
}

Laboratory For Laser Energetics

University of Rochester

250 East River Road

Rochester, New York 14623-1299

and

C. F. HOOPER, JR. and D. A. HAYNES, JR.

Department of Physics, University of Florida, Gainesville, Florida 32611

Q. SU

Department of Physics, Illinois State University, Normal, Illinois 61790-4560

\section{ABSTRACT}

High-temperature laser target implosions can be achieved by using relatively thinshell targets, and they can be diagnosed by doping the fuel with krypton and measuring $\mathrm{K}$-shell and L-shell lines. Electron temperatures of up to $5 \mathrm{keV}$ at modest compressed densities $\left(\sim 1-5 \mathrm{~g} / \mathrm{cm}^{3}\right)$ are predicted for such experiments, with ion temperatures peaking above $10 \mathrm{keV}$ at the center. It is found that the profiles of low-opacity (optically thin) lines in the expected density range are dominated by the Doppler broadening and can provide a measurement of the ion temperature if spectrometers of spectral resolution $\Delta \lambda / \lambda \geq 1000$ are used. For high-opacity lines, obtained with a higher krypton fill pressure, the measurement of the escape factor can yield the $\rho R$ of the compressed fuel. At higher densities, Stark broadening of low-opacity lines becomes important and can provide a density measurement, whereas lines of higher opacity can be used to estimate the extent of mixing. 


\section{INTRODUCTION}

High-temperature laser target implosions can be achieved by using relatively thinshell targets. Electron temperatures of up to $5 \mathrm{keV}$ at modest compressed densities $\left(\sim 1-5 \mathrm{~g} / \mathrm{cm}^{3}\right)$ are predicted for these targets. ${ }^{1}$ The corresponding ion temperatures are predicted to be higher, peaking above $10 \mathrm{keV}$ at the center. The purpose of this paper is to explore the range of diagnostic methods made possible with the krypton doping of the fuel. The emphasis will be on K-shell krypton lines, i.e., transitions to the ground states of the helium-like and hydrogen-like ionic species. Detailed $\mathrm{Kr}$ Stark profile calculations are used. It is found that the profiles of low-opacity (optically thin) lines in the expected range of density are dominated by the Doppler broadening; they can provide a measurement of the ion temperature if spectrometers of spectral resolution $\Delta \lambda / \lambda \geq 1000$ are used. For high-opacity lines, obtained with a higher krypton fill pressure, the measurement of the escape factor can yield the $\rho R$ of the compressed fuel. At higher densities, Stark broadening of low-opacity lines becomes important and can provide a measurement of the density, whereas lines of higher opacity can be used to estimate the extent of mixing. These higher densities stipulate future laser experiments where both high temperatures and high densities will be achieved.

As an example of a simulated high-temperature implosion, we show in Fig. 1 temperature and density profiles (2), at peak compression, calculated by the LILAC code for a $\mathrm{CH}$ shell of $1-\mathrm{mm}$ diameter and $10-\mu \mathrm{m}$ thickness, filled with a 10 -atm pressure of DT. Typical parameters of the OMEGA laser system at the Laboratory for Laser Enegetics were assumed: laser energy of $30 \mathrm{~kJ}$ in a Gaussian pulse of 650 -ps width. As Fig. 1 shows, the core temperature and density are fairly uniform at $\sim 5 \mathrm{keV}$ and $\sim 4.5$ $\mathrm{g} / \mathrm{cm}^{3}$, respectively. In the analysis that follows, the core profiles will be assumed to be uniform. On the other hand, the ion temperature ranges from $\sim 6 \mathrm{keV}$ to $\sim 12 \mathrm{keV}$ and is centrally peaked. The relatively high temperature of the shell enables transmitting core radiation with little attenuation. 
The $\mathrm{K}$-shell lines of $\mathrm{Kr}$ are of much shorter wavelength than most spectral lines measured in current laser-fusion experiments; for example, the $\mathrm{Kr}^{+34}$ resonance line has a wavelength of $0.9455 \AA$ (3), or photon energy of $13.113 \mathrm{keV}$. This line was previously observed in short-pulse (100-ps, 6-TW) experiments (4) on OMEGA, using a VonHamos focusing spectrometer. In recent experiments on the OMEGA system, very intense emission of these krypton lines has been observed (5).

Information on the expected krypton line intensities as well as their contrast with respect to the underlying continuum can be obtained by computer simulation. Figure 2 shows the time-integrated spectrum predicted by the one-dimensional hydrodynamic code LILAC. The target and laser conditions of Fig. 1 were assumed, with the addition of 0.01 atm krypton gas to the fuel. LILAC calculates the $\mathrm{Kr}$ average-ion-level populations by non-LTE rate equations. This calculation does not include a detailed, high-resolution description of the line spectrum but accounts for the gross energetics, such as radiation cooling due to the addition of krypton. In order to obtain higher-resolution spectral results, such as in Fig. 2, a non-LTE post processor is run subsequent to the LILAC run. Here the average-ion model is replaced by actual atomic transitions of various $\mathrm{Kr}$ ionic species, calculated as screened hydrogenic states. Line profiles due to both the Doppler and Stark effects were included. Radiation transport includes photoexcitation and photoionization. For these calculations, only approximate Stark profiles were used; later we show results of detailed calculations of line profiles not included in LILAC. For lowopacity cases, the results such as in Fig. 2 do show the correct total line intensities, even though the line shapes are only approximate.

LILAC calculations show that the addition of 0.01 atm of krypton hardly changes the core temperature. By adding 0.1 atm of krypton, the energy loss due to ionization and radiation causes the peak temperature to drop to about $4 \mathrm{keV}$. For higher amounts of krypton, the peak temperature drops even more significantly. The time-integrated line 
intensities in Fig. 2, of the order of $10^{17} \mathrm{keV} / \mathrm{keV}$, are very substantial; estimates of instrument sensitivities show that measurements with either time-integrating or time-resolving spectrographs should easily record the strong K-shell lines of Fig. 2 under these conditions. Additionally, the contrast of lines to continuum is adequate for measurement; for example, it is better than a factor 4 for the He- $\beta$ line. Furthermore, the shape of the instantaneous spectrum above $\sim 10 \mathrm{keV}$ at peak compression differed little from the time-integrated spectra because most of the emission was emitted at peak compression. Thus, a time-integrated measurement at this spectral range yields the conditions at peak compression with reasonable reliability.

\section{DENSITY DIAGNOSTIC USING LOW-OPACITY LINES}

The diagnostic methods discussed in this paper rely on detailed Stark profile calculations that are used directly as diagnostic signatures or as input to escape-factor calculations. A recently developed, multielectron radiation line profile formalism and code, MERL (6,7), was used to calculate Stark-broadened emission line profiles for the $\mathrm{Kr} \mathrm{K}$-shell lines. The analysis in this paper relies in particular on the helium- $\beta$ transition $\left(3^{1} \mathrm{P}-1^{1} \mathrm{~S}\right)$, and the profiles shown here pertain to this line. Broadening due to perturbing ions is treated in the quasi-static ion approximation, and the dynamic effect of electrons is calculated using a second-order relaxation theory. Ion-and electron-radiator interactions are computed in the dipole approximation. The necessary atomic physics data (energy-level structure and reduced dipole matrix elements) were calculated with Cowan's (8) multi-configuration atomic structure code, using the Hartree-Plus-StatisticalExchange method for approximating the potential energy function due to the electrons, and including relativistic corrections.

Figures 3 and 4 show examples of profile calculations. The Stark profile of the helium $-\beta$ line of $\mathrm{Kr}^{+34}$, of wavelength $0.8033 \AA$, is calculated for two densities: $1 \times 10^{24} \mathrm{~cm}^{-3}$ and $5 \times 10^{24} \mathrm{~cm}^{-3}$. It is assumed that the krypton constitutes a small 
fraction mixed into the fuel and most of the perturbers are fuel ions. The mixing of shell material into the fuel would modify the profiles; however, bacause the nuclear charge of carbon is much smaller than that of krypton, the profiles would depend mainly on the total density and weakly on the exact fuel composition. The effect of Doppler broadening was also calculated, at an assumed ion temperature of $10 \mathrm{keV}$. The shape of the Stark profile is significantly affected by relativistic effects in the atomic physics, due to the relatively high nuclear charge $Z$ of krypton. There are two dipole-allowed transitions from the $1 \mathrm{~s} 3 l$ upper-level manifold to the ground state. The unperturbed singlet-singlet transition is located at $15451 \mathrm{eV}$, and the triplet-singlet transition is located at $15426 \mathrm{eV}$. Additional peaks appear because of field mixing and splitting among the levels of the upper manifold.

Comparison of Figs. 3 and 4 clearly shows the effect of increasing density on the profile. The lower density, $1 \times 10^{24} \mathrm{~cm}^{-3}\left(\sim 4.5 \mathrm{~g} / \mathrm{cm}^{3}\right)$, is at the upper end of the expected density range for the high-temperature target shots. The higher density, $5 \times 10^{24} \mathrm{~cm}^{-3}\left(\sim 22.5 \mathrm{~g} / \mathrm{cm}^{3}\right)$, reflects future experiments where both high density and high temperature will be achieved. For the lower-density case (Fig. 3) the combined profile width is dominated by the Doppler broadening and can thus yield the ion temperature. To measure this width, a spectral resolution $\lambda / \Delta \lambda$ greater than $\sim 1000$ is required. This is not easily achievable with a flat crystal spectrometer, unless employed at a very large distance from the target; however, a Rowland-circle focusing spectrograph can readily achieve this resolution (9). In going from the lower density $\left(1 \times 10^{24} \mathrm{~cm}^{-3}\right)$ to the higher density $\left(5 \times 10^{24} \mathrm{~cm}^{-3}\right)$ the line width more than doubles, reflecting the increasing effect of Stark broadening. It should be noted, however, that this broadening is mostly the result of the increase in intensity of forbidden components. Only at much higher densities will the total width of the manifold be determined by the Stark width rather than by the separation of the components. For the density range covered by Figs. 3 and 4 , density signatures are provided by the width as well as the shape of the compound 
profile, which in turn is determined by the change in intensity and spectral position of the various components. In particular, in going from the lower to the higher density, the peak intensity of the compound profile shifts to lower energies by about $10 \mathrm{eV}$ (due to the socalled "level repulsion" in second-order perturbation theory). To measure this shift, a spectral resolution $\lambda / \Delta \lambda$ greater than $\sim 2000$ is required.

\section{DETERMINATION OF ELECTRON TEMPERATURE}

The electron temperature can be determined from experimental spectra in two ways: by the continuum slope and by the intensity ratio of low-opacity lines. The timeintegrated continuum intensity is sufficiently high to be measured with conventional crystal spectrographs. The time-integrated continuum slope in Fig. 2 is virtually indistinguishable from the time-resolved continuum slope at the time of peak emission. Time-integrated spectral measurement therefore yields the temperature at peak emission (or peak compression). Thus, the slope in Fig. 2 yields $\mathrm{T}_{\mathrm{e}} \sim 4.8 \mathrm{keV}$, which is essentially identical to the volume-averaged temperature in Fig. $1\left(\left\langle\mathrm{~T}_{\mathrm{e}}\right\rangle=4.8 \mathrm{keV}\right)$.

We next calculate the temperature dependence of a particular $\mathrm{Kr}$ line-intensity ratio under steady-state conditions, using the collisional-radiative atomic code POPION (10). These calculations are steady state and non-LTE and do not include photoexcitation or photoionization (but do include radiative recombination and spontaneous emission). To minimize opacity effects we use the following two lines: (a) the Lyman- $\alpha$ line of $\mathrm{Kr}^{+35}$, of wavelength $0.9196 \AA$ and absorption oscillator strength 0.4162 , and (b) the helium- $\beta$ line of $\mathrm{Kr}^{+34}$, of wavelength $0.8033 \AA$ and absorption oscillator strength 0.1293 . We must show that the effect of opacity on these lines will be negligible (i.e., when $\tau<1$ ) for the method to be applicable. We concentrate on the helium- $\beta$ line since the opacity of the Lyman- $\alpha$ line is much smaller. The line opacity at an energy separation $\delta E$ from the unperturbed position can be expressed as [see Eq. (8-15) in Ref. 11] 


$$
\tau(\delta E)=\left(\pi e^{2} h / M m c\right) P(\delta E) f \rho R \varepsilon Q_{n},
$$

where $M$ is the krypton ionic mass, $\mathrm{P}(\delta \mathrm{E})$ is the line profile at $\delta \mathrm{E}$ (in inverse energy units), $f$ is the absorption oscillator strength of the line, $\rho \mathrm{R}$ is the total areal density (mostly that of the fuel), $\varepsilon$ is the fraction of krypton in the fuel (by mass), and $Q_{n}$ is the fraction of krypton ions in the absorbing level (i.e., the lower level of the transition). We assume the addition of 0.01 atm krypton to the DT-filled target and the target conditions of Fig. 1. In that implosion the DT fill pressure was $10 \mathrm{~atm}$, and the total $\rho R$ at peak compression was $\sim 16 \mathrm{mg} / \mathrm{cm}^{2}$. According to the POPION code results, $\mathrm{Q}_{1}$ for heliumlike $\mathrm{Kr}$ and for the temperature range of interest $(\sim 3-10 \mathrm{keV})$ is very close to 1 . The line profile of Fig. 3, relevant to the predictions in Fig. 1, gives $\mathrm{P}(\delta \mathrm{E}=0)=30 \mathrm{keV}^{-1}$. Substituting these values into Eq. (1) yields an opacity of $\tau_{0}=0.43$, hence, smaller than 1. The opacity of the Lyman- $\alpha$ line of $\mathrm{Kr}^{+35}$ is much smaller than that of the helium- $\beta$ line because the ratio of $\mathrm{Kr}^{+35}$ to $\mathrm{Kr}^{+34}$ ground-state populations at $\mathrm{Ne}_{\mathrm{e}}=10^{24} \mathrm{~cm}^{-3}$ varies over the 3- to $10-\mathrm{keV}$ temperature range from $\sim 10^{-3}$ to $\sim 10^{-1}$. Nevertheless, Fig. 2 shows that for a temperature of $\sim 5 \mathrm{keV}$ the Lyman- $\alpha$ line is intense enough to be easily observable.

Figure 5 shows the intensity ratio of the Lyman- $\alpha$ line of $\mathrm{Kr}^{+35}$ to the helium- $\beta$ line of $\mathrm{Kr}^{+35}$, calculated by POPION, as a function of temperature, for two electron density values. The intensity ratio is sensitive to temperature changes, but the Lyman- $\alpha$ line may be too weak to be observed for temperatures smaller than about $\sim 4 \mathrm{keV}$. In going from $\mathrm{T}=10 \mathrm{keV}$ to $\mathrm{T}=4 \mathrm{keV}$, both the ratio in Fig. 5 and the intensity of the helium- $\beta$ line drop by an order of magnitude, which makes the intensity of the Lyman- $\alpha$ line to drop by two orders of magnitude. Over a wide density range (changing by a factor of 20), the temperature-dependence curve changes very little because of the proximity to the corona model, where the line ratio is independent of density. If we know the density 
to be within this range, the maximum error in determining the temperature would be less than $\pm 10 \%$. The required precision in the intensity measurements is modest: to achieve a $\pm 10 \%$ precision in the temperature, the intensity ratio must be measured with a precision of only a factor of $\sim 3$ (at $\mathrm{T}=4 \mathrm{keV}$ ) or a factor of $\sim 2$ (at $\mathrm{T}=6 \mathrm{keV}$ ). The attenuation of these two $\mathrm{Kr}$ lines through the compressed $\mathrm{CH}$ polymer shell is of no concern, as a cold $\rho \Delta R$ of more than $1 \mathrm{~g} / \mathrm{cm}^{2}$ is needed to significantly attenuate them.

It should be noted that dielectronic satellites near the helium-and hydrogen-like lines were not included in the present analysis. These lines, if intense, can compromise the measurement of line intensities as given by Fig. 5. However, POPION steady-state results show that the fractional population of lithium-like ions is small: for $\mathrm{T}=3 \mathrm{keV}$ it is 0.18 , and it drops sharply with increasing temperatures. Thus, the satellite line intensity can be expected to be small. Furthermore, the high-resolution spectrometer which was shown above to be required for line-profile measurements, can help separate the satellites from the adjacent resonance lines.

An additional factor to consider is the effect of opacity on the intensity ratio of Fig. 5. It was shown (12) that high opacity of the helium-like resonance line (in that case, of argon) increases the relative intensity of hydrogen-like lines because of ionization from the upper level of that line. For the krypton fill pressure assumed in Fig. 2 (0.01 atm) we showed that the lines used to derive the temperature in Fig. 5 are optically thin; however, this is not the case for the helium-like resonance line. We defer discussion of this effect to the end of Sec. IV, which deals with opacity.

We can check the usefulness of Fig. 5 as a temperature diagnostic by comparison with the LILAC-calculated spectrum of Fig. 2. The ratio of the Lyman- $\alpha$ to the helium- $\beta$ lines in Fig. 2 is 0.23 . This ratio was obtained by subtracting the underlying continuum and integrating over the expanded line profiles. According to Fig. 5, at a density of $10^{24} \mathrm{~cm}^{-3}$ this ratio corresponds to $\mathrm{T}_{\mathrm{e}}=5.7 \mathrm{keV}$, agreeing closely with the peak core temperature shown in Fig. $1\left(\mathrm{~T}_{\mathrm{e}} \sim 5.5 \mathrm{keV}\right)$. 


\section{IV. $\quad \rho \mathrm{R}$ DIAGNOSTICS USING HIGH-OPACITY LINES}

The opacity of the helium- $\beta$ line of $\mathrm{Kr}^{34+}$ for a fill pressure of 0.01 atm was shown in the previous section to be smaller than $1\left(\tau_{0} \sim 0.55\right)$, and thus negligible. We now examine the case of much higher fill pressures, where the helium- $\beta$ line is optically thick at peak compression. Although the previous analysis is then not applicable, a different type of information on the target behavior can be obtained. Anticipating the following section, we choose the helium- $\beta$ line rather than the helium- $\alpha$ line, which has a higher opacity.

Optically thick spectral lines can be used to deduce the $\rho R$ of the compressed core. The self-absorption of spectral lines (i.e., the absorption by the same transition as that of the emission line) affects both the emergent line intensity as well as its spectral shape. Self-absorption leads to broadening. In the past, the broadening due to selfabsorption of the Lyman- $\alpha$ line of argon was employed to estimate the core $\rho \mathrm{R}$ (13). As explained there, the density must be known (by fitting Stark profiles to an optically thin line) to deduce the $\rho R$ from an optically thick line. Alternatively, the width of several optically thick lines in the same line series must be measured (14). These methods can be employed here as well. However, we pursue an alternative method, based on the intensity of a single optically thick line, rather than its profile; as it turns out, useful information of the fuel $\rho R$ or the state of mixing can be obtained without a prior knowledge of the density or by using additional lines.

The intensity of an optically thick line emerging from the plasma volume is related to the escape factor parameter, which has been the subject of numerous publications (15-18). The escape factor $G$ is defined by 


$$
\mathrm{G}\left(\tau_{0}\right)=\int_{-\infty}^{\infty} \mathrm{P}(\delta \mathrm{E}) \exp \left[-\tau_{0} \mathrm{P}(\delta \mathrm{E}) / \mathrm{P}(\delta \mathrm{E}=0)\right] \mathrm{d}(\delta \mathrm{E})
$$

The spectral position $\delta \mathrm{E}=0$, which is somewhat arbitrary, is taken to be $15449 \mathrm{eV}$, the position of the strongest line for $\mathrm{Ne}_{\mathrm{e}}=1 \times 10^{24} \mathrm{~cm}^{-3}$ (see Fig. 3).

The escape factor $\mathrm{G}\left(\tau_{0}\right)$ in spherical geometry, as given by Eq. (2), corresponds to a point source at the center of the sphere, and $\tau_{0}$ is the opacity over the radius. For a source uniformly distributed over the sphere, Mancini et al. (18) have shown that $\mathrm{G}\left(\tau_{0}\right)$ for Holtsmarkian profiles and large opacities is about twice as large as in the point-source case. This indicates that knowing the temperature profile (or spatial distribution of emitting and absorbing ions) is not critical when using the calculated escape factor for diagnostics. Thus, a two-temperature spatial profile (hot spot surrounded by a cool absorbing layer) and a uniform temperature profile give an escape factor that differs by only a factor of 2 for any $\tau_{0} \gg 1$. To determine which geometry agrees better with the experiment we can examine two experimental signatures: (a) for a uniform source, the core image size at high photon energy will be about the same as that at low photon energy, whereas for a hot-spot source the former will be much smaller than the latter, and (b) for a uniform source the observed line profile will be flat topped, whereas for a hotspot source a self-reversal (or minimum) will be observed at the position of the profile peaks. Figure 1 shows that the expected spatial distributions should be closer to the uniform case. Equation (2) applies to a two-temperature spatial profile but assumes that the line profile is the same in both the emission and absorption regions. This implies a uniform density since the density-dependent Stark broadening dominates the emergent profile for high opacities (see below). As an example, if the absorption line profile is half as wide as the emission profile (indicating a lower density by about a factor of 2.8 ), the escape factor can be shown to be larger by about $30 \%$.

The asymptotic behavior of the escape factor for $\tau_{0} \gg 1$ depends primarily on the type of line profile (15). For example, for a Gaussian (i.e., Doppler) profile, $G\left(\tau_{0}\right)$ does 


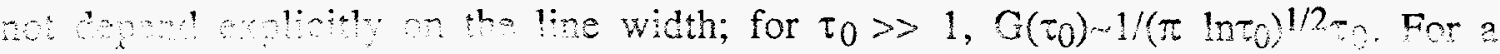

Holtzmarkian profile (the simplest approximation to a Stark profile) the asymptotic expression $(15)$ is $\mathrm{G}\left(\tau_{0}\right) \sim 0.451 / \tau_{0} 3 / 5$.

To obtain the escape factor $\mathrm{G}\left(\tau_{0}\right)$ from $\mathrm{Eq}$. (2) for our case, we use profiles such as in Figs. 3 and 4. Figure 6 shows the escape factor as a function of the opacity at line center $(15449 \mathrm{eV})$, with and without the Doppler profile contribution. The curves are calculated for an ion temperature of $10 \mathrm{keV}$. The escape factor $\mathrm{G}$ depends on the temperature only through the Doppler contribution. Figure 6 shows that we can almost eliminate this dependence by plotting $\mathrm{G}$ as a function of $\tau_{0, S}$, the opacity at line center due to the Stark profile alone. When changing from $\tau_{0}$ to $\tau_{0, S}$, the escape factor curves for the total profile shifts to opacity values that are larger (since $\tau_{0, S}>\tau_{0}$ ), by the ratio $\tau_{0, S} / \tau_{0}$. The two curves (escape factor with and without the Doppler effect, as a function of $\left.\tau_{0, S}\right)$ are almost coincident. The reason for this is simple: The Doppler profile decays much faster, with increasing separation from line center, than the Stark profile. Thus, the far wings are relatively unaffected by the inclusion of the Doppler effect. However, the escape factor for high opacity values depends mostly on the far wings (see Eq. 2). Therefore, the escape factor for high opacities will be relatively independent of the temperature. Since the profile on the far wings is essentially identical to the Stark profile, the escape factor will depend only on the Stark profile at line center.

A similar situation will arise if we were to include ion-dynamic effects in the calculation of the Stark profiles. Effects of the motion of perturbing ions on the Stark profile (which were assumed stationary) will depend also on the ion temperature and will affect mostly the line center rather than its wings. Thus, the opacity $\tau_{0, \mathrm{~S}}$ relates to the Stark profile without either the Doppler effect or the ion-dynamic effects.

Comparison of profiles in Fig. 6 shows also the relative insensitivity of the escape factor to the density. As mentioned earlier, for a Holtsmarkian profile and high opacities, $\mathrm{G}\left(\tau_{0}\right)$ depends only on $\tau_{0}$ and not on the density (or the line width). The escape factor is 
sometimes expressed (18) in terms of $\tau_{0} / \mathrm{P}(\delta \mathrm{E}=0)$, in which case the Holtzmark mean field $F_{0}$ also appears. The density dependence of $\mathrm{P}(\mathrm{\partial E}=0)$ and $\mathrm{F}_{0}$ cancels out for a Holtzmark profile (and approximately for other Stark profiles). For easier comparison, Fig. 7 shows the escape factor curves from Figs. 6(a) and 6(c), calculated without the Doppler contribution, for the two densities: $1 \times 10^{24} \mathrm{~cm}^{-3}$ and $5 \times 10^{24} \mathrm{~cm}^{-3}$. As seen, the two profiles overlap to within a factor of $\sim 1.4$. Similar agreement is obtained in comparing curves that do include the Doppler broadening. We can thus use Fig. 6 to find the quantity $\tau_{0, S}$ from a measured value of the escape factor, without a precise knowledge of the temperature or the density. The question before us now is what does the opacity $\tau_{0, S}$ depend on, i.e., what diagnostic information can be inferred from knowing $\tau_{0, \mathrm{~s}}$.

From Fig. 6, the escape factor for high opacity, in the density range $(1-5) \times 10^{24} \mathrm{~cm}^{-3}$, can be approximated by the relation

$$
\mathrm{G}\left(\tau_{0, \mathrm{~S}}\right) \sim 1.3 /\left(\tau_{0}, \mathrm{~S}\right)^{0.9}
$$

Only at much higher densities, where level mixing of the $n=3$ manifold due to the plasma electric field becomes substantial, will this relationship tend to that for an Holtsmarkian profile mentioned above. That case is discussed in the following section. For the density range discussed here, Eq. (3) leads to a dependence of $\tau_{0, S}$ on the density (or on the $\rho \mathrm{R}$ ), thus providing a diagnostic for $\rho \mathrm{R}$.

The diagnostic method for the fuel $\rho R$ consists of measuring the escape factor as outlined above, then deducing $\tau_{0,5}$ from curves like those in Fig. 6 . It follows from the definition of $\tau_{0}$ [Eq.(1)] that if we substitute $\tau_{0, S}$ for $\tau_{0}$, the profile value $\mathrm{P}_{S}(\delta \mathrm{E}=0)$ appearing in Eq. (1) should refer to the value of the pure Stark profile, $P_{S}(\delta E=0)$. $P_{S}(\delta E=0)$ can be read off the calculated Stark profiles, such as in Figs. 3 and 4, and is 
obviousily a function of the density. In the range $(1-5) \times 10^{24} \mathrm{~cm}^{-3}, \mathrm{P}_{S}(\delta E=0)$ as a function of the density can be approximated as

$$
\mathrm{P}_{\mathrm{S}}(\delta \mathrm{E}=0) \sim 0.4 / \rho^{0.88}
$$

In any practical case, the complete profile curves can be used rather than this approximate expression. Further, in an imploding spherical target the following relationship holds:

$$
\rho R=\left(3 M_{F} / 4 \pi\right)^{1 / 3} \rho^{2 / 3},
$$

in terms of the total fill mass $M_{F}$ (fuel and krypton). Combining Eqs. (1), (3), (4), and (5) we can write

$$
\rho R=0.02 A^{3.125}\left(3 \mathrm{M}_{F} / 4 \pi\right)^{1.375} \mathrm{G}^{3.437}
$$

where $A=\left(\pi e^{2} h / M m c\right) f \varepsilon Q_{n}$ [from Eq. (1)]. As mentioned above, by using the POPION code, $\mathrm{Q}_{\mathrm{n}}$ was shown to be very close to unity over a wide temperature range. This relation is the basis for determining the fuel $\rho R$ from a measurement of the escape factor G. As mentioned above, actually calculated curves can be used rather than the approximations given in Eqs. (3) and (4). A method for measuring the escape factor is discussed in the final section.

It was mentioned above that the line-intensity ratio used in Fig. 5 to derive the temperature can also depend on opacity effects. We can now estimate this effect. For the krypton fill pressure assumed in Fig. 2 (0.01 atm) we showed in Sec. III that the lines used to derive the temperature in Fig. 5 (namely, the helium- $\beta$ and Lyman- $\alpha$ lines) are optically thin for the target implosion of Fig. 1. However, this is not the case for the helium-like resonance line. The opacity of this line for the conditions of Fig. 1, using 
Eq. (1), can be estimated to be in the range 5-10. This opacity increases the line-intensity ratio indirectly (12), by increasing the ionization rate from $\mathrm{Kr}+34$ to $\mathrm{Kr}+35$. This increase is due to ionization from the $\mathrm{n}=2$ manifold of $\mathrm{Kr}^{+34}$, whose population is enhanced by self-absorption of the $1 \mathrm{~s}^{2}-1 \mathrm{~s} 2 \mathrm{p}^{1} \mathrm{P}$ transition in $\mathrm{Kr}^{+34}$. Figure 5 is based on the POPION atomic code (10), which does not include opacity effects; thus, the rate $\left(\mathrm{per} \mathrm{cm}^{3}\right)$ for ionizing $\mathrm{Kr}^{+34}$ ions is given by $\mathrm{N}_{\mathrm{e}} \mathrm{N}_{1} \mathrm{~S}_{1}(\mathrm{~T})$, where $\mathrm{N}_{\mathrm{e}}$ is the electron density, $\mathrm{N}_{1}$ is the density of $\mathrm{Kr}^{+34}$ ions in the ground state, and $\mathrm{S}_{1}(\mathrm{~T})$ is the ionization rate coefficient. The increase factor $R_{i}$ in this rate (and thus in the line-intensity ratio of Fig. 5) due to the helium- $\alpha$ opacity is given by

$$
R_{i}=1+\left[N_{2} S_{2}(T) / N_{1} S_{1}(T)\right]
$$

The ratio of the ionization coefficient from the $n=2$ manifold (excitations to the $1 \mathrm{~s}^{2} \mathrm{p}^{1} \mathrm{P}$ level are distributed to other $\mathrm{n}=2$ levels) to that from the ground level can be estimated using Ref. 19. The effect of opacity on line emission can be expressed as a reduction in the Einstein A coefficient for spontaneous emission by a factor equal to the escape factor (15). Thus, the population of the upper level will be enhanced by the same factor due to the opacity of the resonance line. We can thus write the population ratio $N_{2} / N_{1}$ as $\left[\mathrm{G}\left(\tau_{0}\right)\right]^{-1}\left(\mathrm{~N}_{2,0} / \mathrm{N}_{1,0}\right)$, in terms of the populations for the case where opacity is neglected. The ratio $\left(\mathrm{N}_{2,0} / \mathrm{N}_{1,0}\right)$ is calculated by the POPION code. For the estimated range of opacities of the helium- $\alpha$ line (namely, 5-10) the range of the escape factor for various profiles (16) is in the range of $0.1-0.2$. Finally, Fig. 8 shows the line-intensity ratio plotted in Fig. 5, modified by the effect of opacity of the helium- $\alpha$ line, for $\mathrm{N}_{\mathrm{e}}=1 \times 10^{24} \mathrm{~cm}^{-3}$. Thus, to measure the temperature accurately, a knowledge of the opacity, or the $\rho R$, is required. However, for the conditions expected in the hightemperature experiments studied here, when ignoring opacity the deduced temperature is too high by, for example, $\sim 10 \%$ at $\mathrm{T}=5 \mathrm{keV}$. 


\section{DIAGNOSIS OF SHELL-FUEL MIXING BASED ON HIGH-OPACITY}

\section{Kr LINES}

When the density increases much above $\sim 10 \mathrm{~g} / \mathrm{cm}^{-3}$, such that the mixing of atomic levels due to the plasma electric field dominates the intrinsic atomic splitting, the helium- $\beta$ line behaves as a hydrogenic line, allowing the use of a hydrogenic approximation to the profile calculation. The relations given by Eqs. (3), (4), and (6) would then change. The Stark profile on the far wings, for hydrogen-like lines, is approximately Holtsmarkian (15), for which the corresponding escape factor for $\tau_{0} \gg>1$ was found to be given by

$$
\mathrm{G}\left(\tau_{0}\right) \sim 0.451 / \tau_{0}^{3 / 5}\left(\tau_{0} \gg 1\right)
$$

This expression replaces the lower-density asymptotic relation given by Eq. (3). It will apply to implosions where the density is much higher than $5 \times 10^{24} \mathrm{~cm}^{-3}$, while the temperature is still high enough to excite $\mathrm{Kr} \mathrm{K}$-shell lines. In the hydrogenic limit, since the line width is proportional to $\rho^{2 / 3}(20)$, it follows that

$$
P_{S}(\delta E=0)=\alpha \rho^{-2 / 3} \text {, }
$$

where $\alpha$ is a constant depending on the spectral line. From Fig. 4 it can be shown that $\alpha$ for the helium $\beta$ line is of the order of $\sim 0.5$, if $\mathrm{P}_{\mathrm{S}}(\delta \mathrm{E}=0)$ is expressed in $\mathrm{eV}^{-1}$ and $\rho$ in $\mathrm{g} / \mathrm{cm}^{3}$. At high densities, this relationship replaces the one given by Eq. (4).

Combining Eqs. (1), (5), and (9), we see that $\tau_{0}$ is independent of target compression and depends only on the total fill mass because the opacity increases with increasing $\rho R$, but decreases due to the increasing width (or decreasing $P_{S}$ ). Both quantities change as $\rho^{2 / 3}$ and cancel each other's effect on $\tau_{0}$.

The foregoing discussion shows that measuring the escape factor under the present conditions cannot yield information on the density or $\rho \mathrm{R}$. However, mixing shell 
material into the fuel does affect the escape-factor measurement because the $\rho R$ of krypton alone determines the absorption of $\mathrm{Kr}$ lines, whereas the Stark profile depends on the densities of all species present, including the shell material mixed into the fuel. The total Stark width for the case of a mixture is approximately proportional to the sum $\Sigma Z_{\mathrm{p}} \mathrm{N}_{\mathrm{p}}^{2 / 3}$ over the perturbing species. This dependence appears in the perturbing-ion contribution to the Stark effect of hydrogenic lines (20), which dominates the wings at sufficiently high densities. For the case of a mixture of deuterium ions plus a fraction $\xi$ of stripped carbon ions from the shell $\left(\xi=\mathrm{n}_{C} / \mathrm{n}_{\mathrm{D}}\right)$, the line width at a given total mass density would scale with $\xi$ as $\phi=\left(1+6 \xi^{2 / 3}\right) /(1+6 \xi)^{2 / 3}$, so that Eq. (9) is now replaced by

$$
\mathrm{P}_{\mathrm{S}}(\delta \mathrm{E}=0)=(\alpha / \phi) \rho^{-2 / 3}
$$

Combining Eqs. (1), (5), and (10), we can relate the volume-averaged fraction of density due to mixing $\xi_{\mathrm{m}}=\rho_{\mathrm{mix}} / \rho_{\mathrm{F}} \sim 6 \xi$ to $\tau_{0}$ :

$$
\left(1+1.8 \xi_{\mathrm{m}}\right)^{3 / 2}=\left[\pi \mathrm{e}^{2} \mathrm{~h} \alpha f \mathrm{Q}_{1}\left(3 \mathrm{M}_{\mathrm{F}} / 4 \pi\right)^{1 / 3} /\left(\mathrm{mMc} \tau_{0}\right)\right]^{3 / 2}
$$

We can understand the effect of mixing on the opacity as follows: without mixing, the opacity $\tau_{0}$ is approximately constant during the compression because of the two opposing effects: increase in the $\rho R$ of absorbing ions and increase in the line width. The mixed shell material is involved only in the second effect, which causes a net reduction in the opacity; thus, the higher the mixing, the lower the measured opacity.

The experimental determination of the mixing fraction consists of measuring the escape factor $G\left(\tau_{0}\right)$, deducing $\tau_{0}$ from Eq. (8), or from a calculated curve such as in Fig. 6, and finally finding $\xi$ from Eq. (11). Equation (11) is correct only for a point source 
(central hot spot). As mentioned above, for a spherically uniform source, $G\left(\tau_{0}\right)$ for Holtsmarkian profiles and large opacities is about twice as large (18), and several experimental signatures can be used to estimate deviation from either limit. A method of measuring the escape factor is discussed below.

\section{A METHOD FOR MEASURING THE ESCAPE FACTOR}

A method for measuring the escape factor of a line consists of comparing its measured intensity to that of another line, both of which have the same upper level. The first should have an opacity $\tau_{0} \gg 1$, the second, $\tau_{0} \ll 1$. The two helium-like $\mathrm{Kr}$ lines we selected are (a) the helium- $\beta$ line, $1 \mathrm{~s} 3 \mathrm{p}^{1} \mathrm{P}-1 \mathrm{~s}^{2}{ }^{1} \mathrm{~S}$ (at $0.8033 \AA$ ) and (b) the Balmer- $\alpha$ line, $1 \mathrm{~s} 3 \mathrm{p}^{1} \mathrm{P}-1 \mathrm{~s} 2 \mathrm{~s}^{1} \mathrm{~S}$ (at $5.0508 \AA$ ). Note that what we refer to here as Balmer- $\alpha$ is the helium-like 3-2 transition that shares an upper level with the helium- $\beta$ line (and not, for example, to the stronger $1 \mathrm{~s} 3 \mathrm{~d}^{1} \mathrm{D}-1 \mathrm{~s}^{2} 1 \mathrm{P}$ transition at $5.3463 \AA$ ). For krypton ions, both the transitions to the ground level and the 3-2 transitions are easily accessible to $x$-ray measurement. For argon, the 3-2 transitions are too soft $(\lambda>20 \AA)$ for common $x$-ray crystal instruments, and they also suffer very high opacity in traversing the target.

By making an appropriate choice of the krypton fill pressure, the opacity (for resonant absorption) of the helium- $\beta$ line at peak compression will be $>>1$, while that of the Balmer- $\alpha$ will be $<<1$. It was estimated above that for a $\mathrm{Kr}$ fill pressure of $0.01 \mathrm{~atm}$, the opacity $\tau_{0}$ of the helium- $\beta$ line at peak compression will be $\sim 0.5$. Thus, for a fill pressure in the range $0.1-0.4 \mathrm{~atm}, \tau_{0}$ will be in the range of 5-20; self-consistent code calculations show that the peak electron temperature will be higher than $3 \mathrm{keV}$ and the $\mathrm{Kr}$ $\mathrm{K}$-lines will still be visible. The opacity of the Balmer- $\alpha$ line will still be negligible since it is absorbed by ions in the $n=2$ shell (whereas the helium- $\beta$ line is absorbed by ground-level ions). POPION (10) code calculations show that the population of $n=2$ absorbing ions is smaller than that of $n=1$ absorbing ions by several orders of magnitude. 
In the absence of any absorption, the intensity ratio of these two lines, $\mathrm{I}_{\mathrm{Ly}} / \mathrm{I}_{\mathrm{Ba}}$, is simply given by the ratio of the Einstein A coefficients (spontaneous emission probabilities) $A_{L y} / A_{B a}$ and is independent of any atomic modeling. The required coefficients were calculated (21) as A(helium- $\beta)=4.453 \times 10^{14} \mathrm{~s}^{-1}$ and $\mathrm{A}($ Balmer- $\alpha)=$ $6.163 \times 10^{12} \mathrm{~s}^{-1}$; hence, $\mathrm{A}_{\mathrm{Ly}} / \mathrm{A}_{\mathrm{Ba}}=72.25$. In the case discussed here, the observed intensity ratio $I_{L y} / I_{B a}$ will be lower than the ratio of the Einstein A coefficients $A_{L y} / A_{B a}$, by the escape factor $G$ for the helium- $\beta$ line. Thus, $G$ can be found from

$$
\mathrm{G}=\left(\mathrm{I}_{\mathrm{Ly}} / \mathrm{I}_{\mathrm{Ba}}\right) /\left(\mathrm{A}_{\mathrm{Ly}} / \mathrm{A}_{\mathrm{Ba}}\right)=\left(\mathrm{I}_{\mathrm{Ly}} / \mathrm{I}_{\mathrm{Ba}}\right) / 72.25
$$

It should be noted that the emergent intensity of a high-opacity line may not depend uniquely on the escape factor because of the possibility of re-emission of absorbed photons (22). This is equivalent to allowing for the increased excited-level population (and thus emission) due to the absorption itself. In our case this effect is already included in the ratio of line intensities because the measured intensity of the optically thin Balmer- $\alpha$ line does reflect the actual excited-level population.

The nonresonant absorption by the target material (mostly the shell) should be negligibly small to insure the validity of this method. The attenuation of the helium- $\beta$ line through the shell is negligible: it takes a $\rho \Delta \mathrm{R}$ of $\sim 1.8 \mathrm{~g} / \mathrm{cm}^{2}$ of cold $\mathrm{CH}$ to attenuate that line by $1 / \mathrm{e}$. On the other hand, the Balmer- $\alpha$ line will be attenuated by the same amount in going through only a $\rho \Delta R$ of $\sim 5.5 \mathrm{mg} / \mathrm{cm}^{2}$ of cold $\mathrm{CH}$. However, Fig. 1 shows that the shell at peak compression is hot enough to minimize this attenuation. The opacity of the $\mathrm{CH}$ shell at a wavelength $\lambda$, due to inverse bremsstrahlung absorption, is given by (23) $\tau=2.23 \times 10^{-3} \lambda^{3}(\rho \Delta R) \rho / T^{1 / 2}$, where $\lambda$ is in Angstroms and $T$ in keV. For the target profiles of Fig. 1, the inverse bremsstrahlung opacity is $\tau \sim 0.014$. The opacity of $\mathrm{CH}$ due to photoionization is given by (23) $\tau=0.54 \lambda^{3}(\rho \Delta R) \psi$, where $\psi$ is the fraction of 
carbon ions that are not stripped. POPION results show that for the values of shell temperature and density, $\psi<10^{-3}$, so that the, photoionization opacity is $\tau<10^{-3}$.

Finally, we estimate the expected sensitivity of measuring the compressed fuel $\rho R$ and the degree of shell-fuel mixing. To find G from Eq. (12) with a precision of, say, $\pm 20 \%$, the relative intensity of each of the lines must be measured with a precision of $\pm 10 \%$, which requires the relative calibration of two instruments for the two very different wavelengths used here. A suitable calibration procedure is as follows: the intensity ratio $\mathrm{I}_{\mathrm{Ly}} / \mathrm{I}_{\mathrm{Ba}}$ for the case of a very low $\mathrm{Kr}$ fill pressure $(\sim 0.01$ atm) is simply given by the known ratio $\mathrm{A}_{\mathrm{Ly}} / \mathrm{A}_{\mathrm{Ba}}$; this known intensity ratio calibrates the relative sensitivities of the two spectrometers. From Eq. (3), an error of $\pm 20 \%$ in $G$ will result in an error of $\pm 22 \%$ in $\tau_{0, S}$. Finally, from Eq. (6), the precision in determining the compressed fuel $\rho \mathrm{R}$ will be better than a factor of 2 . For high-density cases, $\mathrm{G}$ depends

asymptotically on $\tau_{0, S}$, like $1 / \tau_{0, S^{3 / 5}}$; thus an error of $\pm 20 \%$ in $\mathrm{G}$ will result in an error of $\pm 33 \%$ in $\tau_{0, S}$. Finding the relative mixing from Eq. (11), this error translates into an error of $\pm 50 \%$ in $1+\xi$. Thus, the method is useful only for extensive mixing, where $\xi$ is not much smaller than 1 .

\section{ACKNOWLEDGMENT}

This work was supported by the U.S. Department of Energy Office of Inertial Confinement Fusion under Cooperative Agreement No. DE-FC03-92SF19460, the University of Rochester, and the New York State Energy Research and Development Authority. The support of DOE does not constitute an endorsement by DOE of the views expressed in this article.

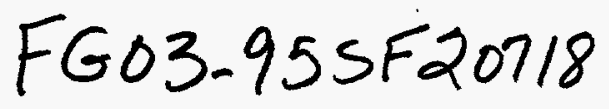




\section{REFERENCES}

1. LLE Review 55, 179 (1993).

2. LLE Review 61, 1 (1994).

3. U. I. Safranova, Phys. Scr. 23, 241 (1981).

4. B. Yaakobi and A. J. Burek, IEEE J. Quantum Electron. QE-19, 1841 (1983).

5. LLE Review 64, 145 (1995).

6. L. A. Woltz and C. F. Hooper, Jr., Phys. Rev. A 38, 4766 (1988).

7. R. C. Mancini, D. P. Kilcrease, L. A. Woltz, and C. F. Hooper, Jr., Comp. Phys. Comm. 63, $314(1991)$.

8. R. D. Cowan, The Theory of Atomic Structure and Spectra (University of California Press, Berkeley, CA, 1981).

9. J. C. Kieffer, M. Chaker, H. Pepin, D. M. Villeneuve, J. E. Bernard, and H. A. Baldis, Appl. Opt. 28, 4333 (1989); B. Yaakobi, T. Boehly, and P. Audebert, Rev. Sci. Instrum. 61, 1915 (1990).

10. R. Epstein, S. Skupsky, and J. Delettrez, J. Quant. Spectrosc. Radiat. Transfer 35, 131 (1986).

11. H. R. Griem, Plasma Spectroscopy (McGraw-Hill, New York, 1964).

12. C. J. Keane, B. A. Hammel, D. R. Kania, J. D. Kilkenny, R. W. Lee, A. L. Osterheld, L. J. Suter, R. C. Mancini, C. F. Hooper, and N. D. Dalamater, Phys. Fluids B 5, 3328 (1993).

13. B. Yaakobi, S. Skupsky, R. L. McCrory, C. F. Hooper, H. Deckman, P. Bourke, and J. M. Soures, Phys. Rev. Lett. 44, 1072 (1980).

14. N. D. Delameter, C. F. Hooper, Jr., R. F. Joyce, L. A. Woltz, N. M. Ceglio, R. L. Kauffman, R. W. Lee, and M. C. Richardson, Phys. Rev. A 31, 2460 (1985).

15. F. E. Irons, J. Quant. Spectrosc. Radiat. Transfer 22, 1 (1979).

16. J. P. Apruzese, Spectrosc. Radiat. Transfer 34, 447 (1985). 
17. C. Chenais-Popovics, P. Alaterre, P. Audebert, J. P. Geindre, and J. C. Gauthier, Spectrosc. Radiat. Transfer 36, 355 (1986).

18. R. C. Mancini, R. F. Joyce, and C. F. Hooper, Jr., J. Phys. B: At. Mol. Phys. 20, 2975 (1987).

19. R. C. Elton, in Methods of Experimental Physics (Academic Press, NY, 1970), Vol. 9A, Sec. 4.3.3.

20. H. R. Griem, in Spectral Line Broadening by Plasmas (Academic Press, New York, 1974), p. 8.

21. Marcel Klapisch (private communication).

22. A. G. Hearn, Proc. Phys. Soc. 81, 648 (1963).

23. B. Yaakobi, R. Epstein, and F. J. Marshall, Phys. Rev. A 44, 8429 (1991). 


\section{FIGURE CAPTIONS}

FIG. 1 Electron temperature, ion temperature, and mass density at peak compression, predicted by the LILAC code for a DT-filled, high-temperature implosion on the OMEGA system. The vertical line marks the fuel-shell interface.

FIG. 2 Time-integrated spectrum calculated by LILAC for the target experiment of Fig. 1, with the addition of 0.01 atm of krypton gas to the fuel.

FIG. 3 Calculated Stark profile of the He- $\beta$ line of $\mathrm{Kr}^{+34}$ (solid line) and the combined Stark and Doppler profile corresponding to an ion temperature of $10 \mathrm{keV}$ (dashed line). An electron density of $1 \times 10^{24} \mathrm{~cm}^{-3}$ was assumed.

FIG. 4 Calculated Stark profile of the He- $\beta$ line of $\mathrm{Kr}^{+34}$ (solid line) and the combined Stark and Doppler profile corresponding to an ion temperature of $10 \mathrm{keV}$ (dashed line). An electron density of $5 \times 10^{24} \mathrm{~cm}^{-3}$ was assumed.

FIG. 5 Intensity ratio of the Lyman- $\alpha$ line of $\mathrm{Kr}^{+35}(0.9196 \AA)$ to the helium- $\beta$ line of $\mathrm{Kr}^{+34}(0.8033 \AA)$ as a function of temperature at two electron-density values. The opacity of both lines, which was shown to be small, was neglected.

FIG. 6 Calculated escape factor of the He- $\beta$ line of $\mathrm{Kr}^{+34}$ for two electron densities, as a function of the opacity at line center $(15451 \mathrm{eV})$, without the Doppler contribution (solid curve) and with the Doppler contribution (dashed curve). In (a) and (c) the opacity $\tau_{0}$ at $15451 \mathrm{eV}$ relates to the total Stark and Doppler 
profile, while in (b) and (d) the opacity $\tau_{0, S}$ at $15451 \mathrm{eV}$ relates to the Stark profile only.

FIG. 7 Calculated escape factor of the He- $\beta$ line of $\mathrm{Kr}^{+34}$ for two electron densities, as a function of the opacity at line center $(15451 \mathrm{eV})$, without the Doppler contribution.

FIG. 8 Intensity ratio of the Lyman- $\alpha$ line of $\mathrm{Kr}^{+35}(0.9196 \AA)$ to the Helium- $\beta$ line of $\mathrm{Kr}^{+34}(0.8033 \AA)$ as a function of temperature, at the electron density of $1 \times 10^{24} \mathrm{c} \mathrm{m}^{-3}$. The solid curve neglecs opacity effects. The dashed curve includes the effect of the opacity of the Helium- $\alpha$ resonance line (assumed to be 5). 


\section{Temperature and density profiles of a high-temperature implosion}

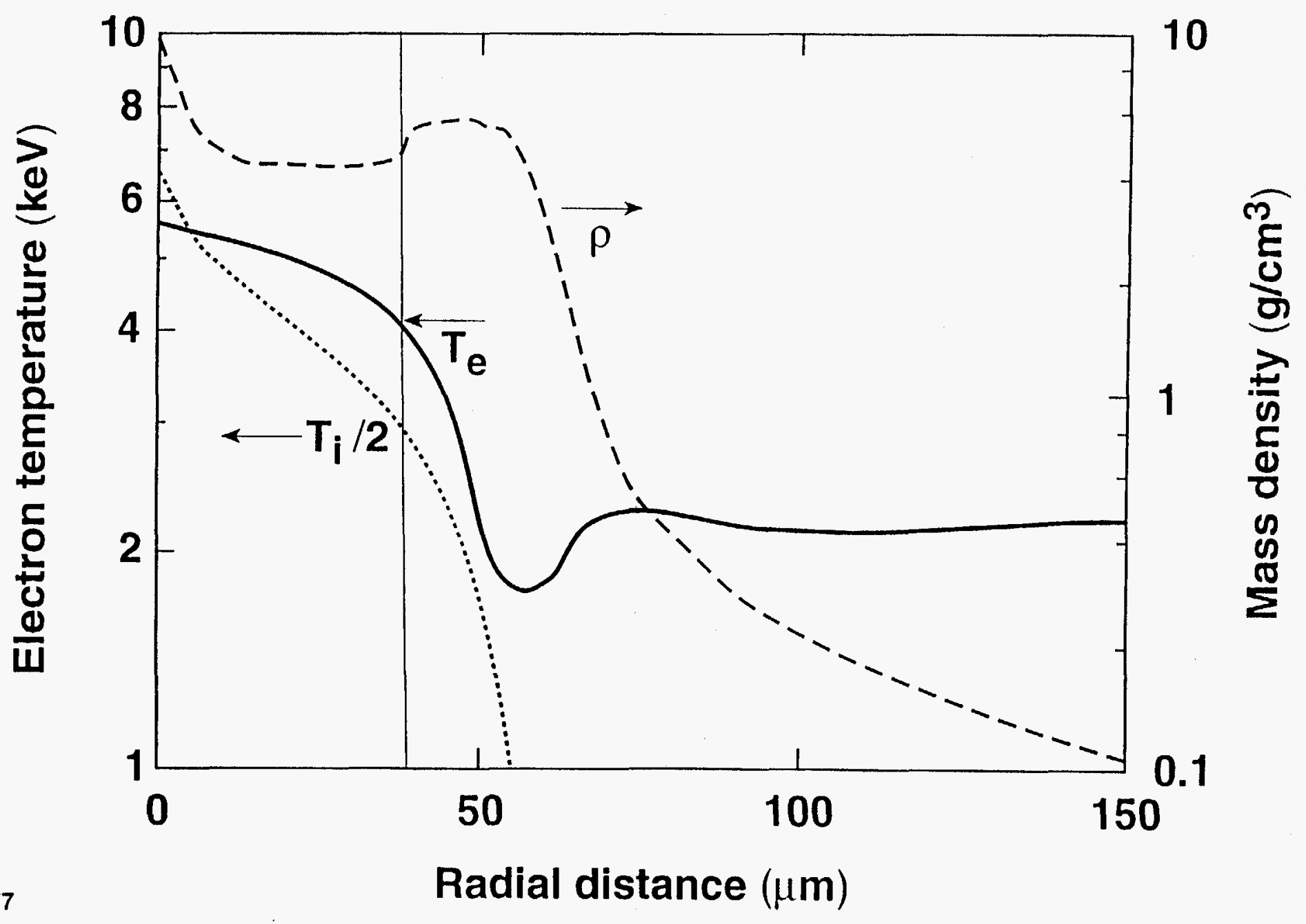




\section{LILAC-calculated spectrum from $\mathrm{Kr}$-doped, high-T implosion}

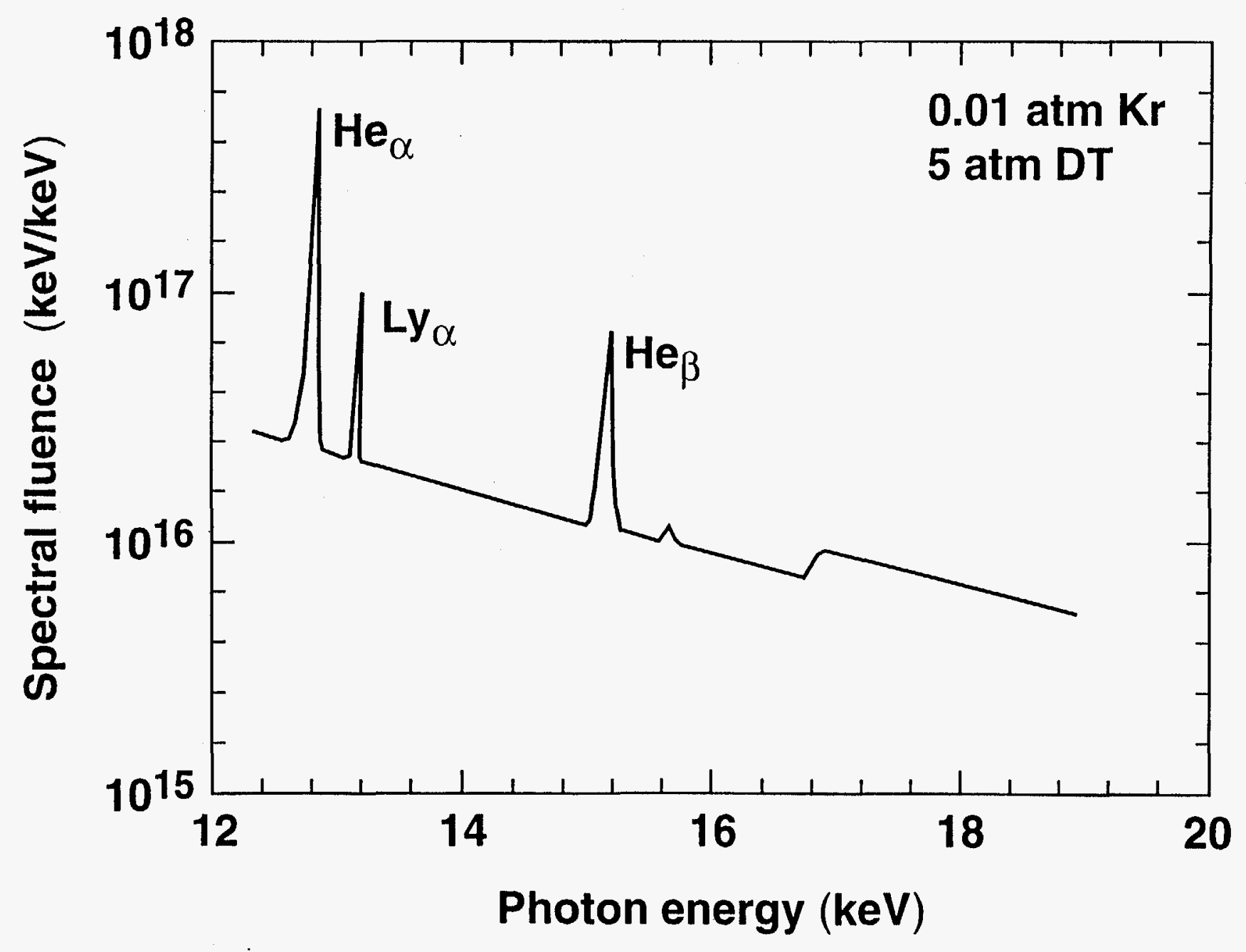




\section{Effect of Doppler broadening on Stark profile}

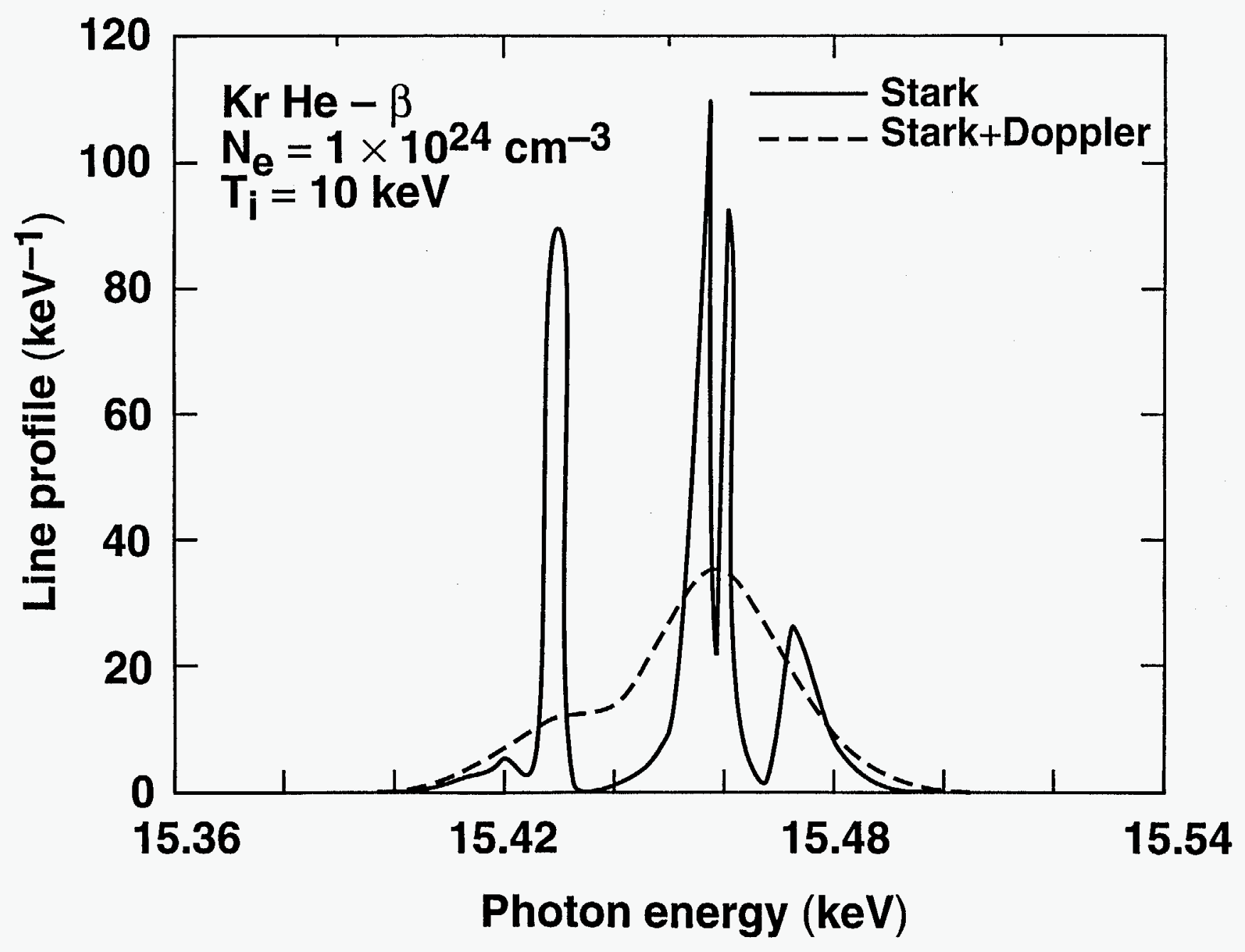




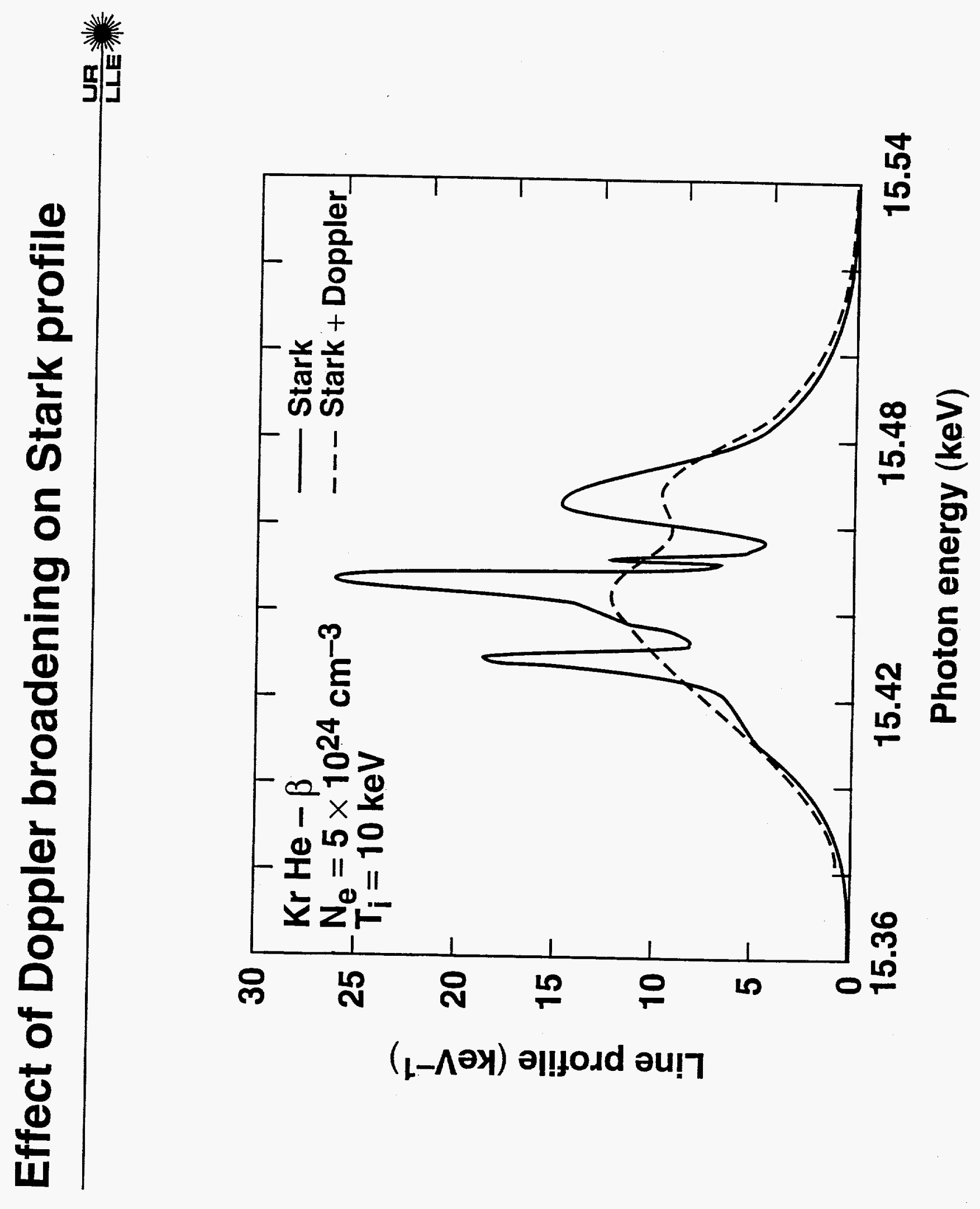

욤

Fig. 4 


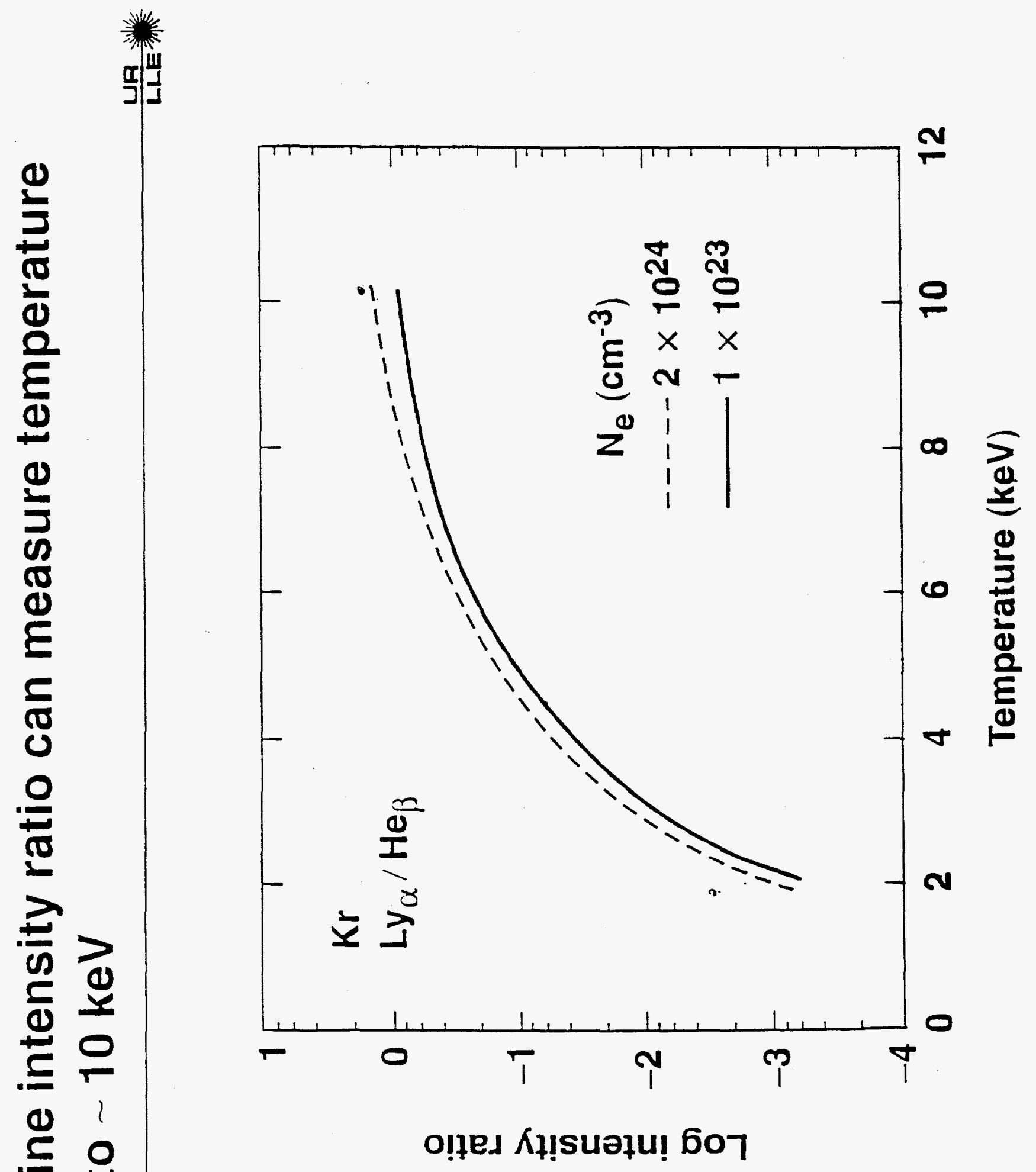

Fig. 5 


\section{Escape factor as a function of $\tau_{0, S}$ is quasi-independent of temperature}
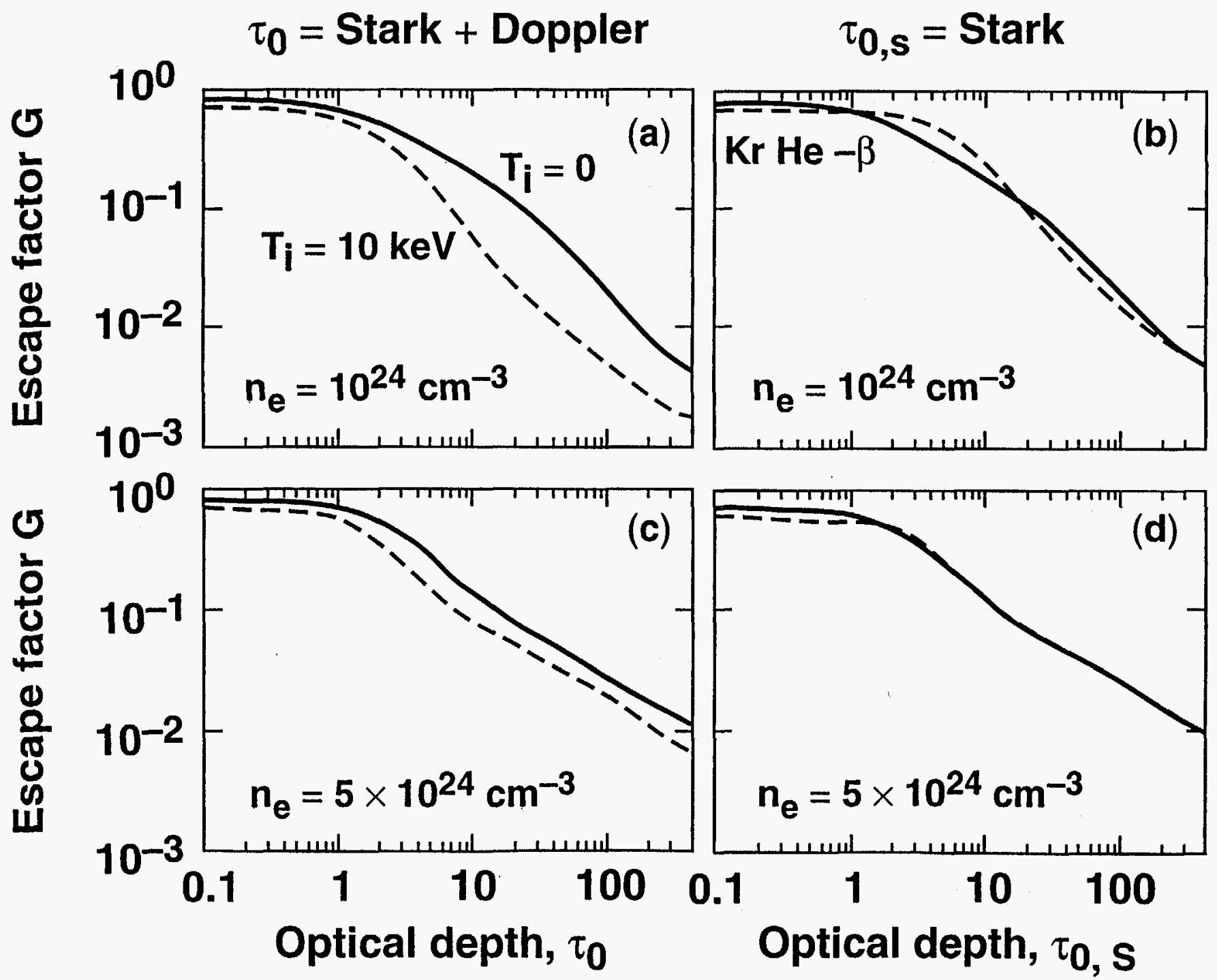
Escape factor for Stark profiles is quasi-independant of density

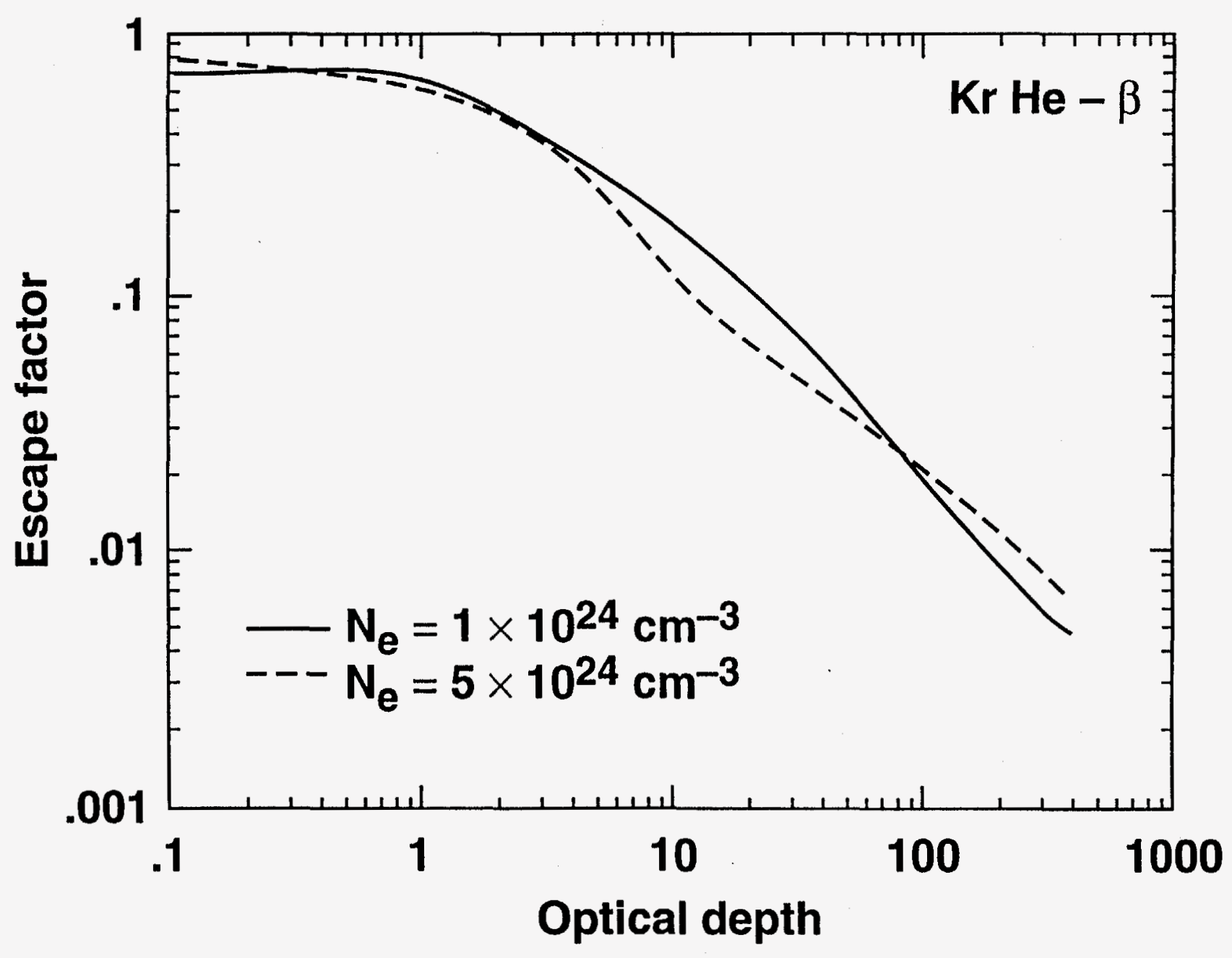


$\because e^{x+}$

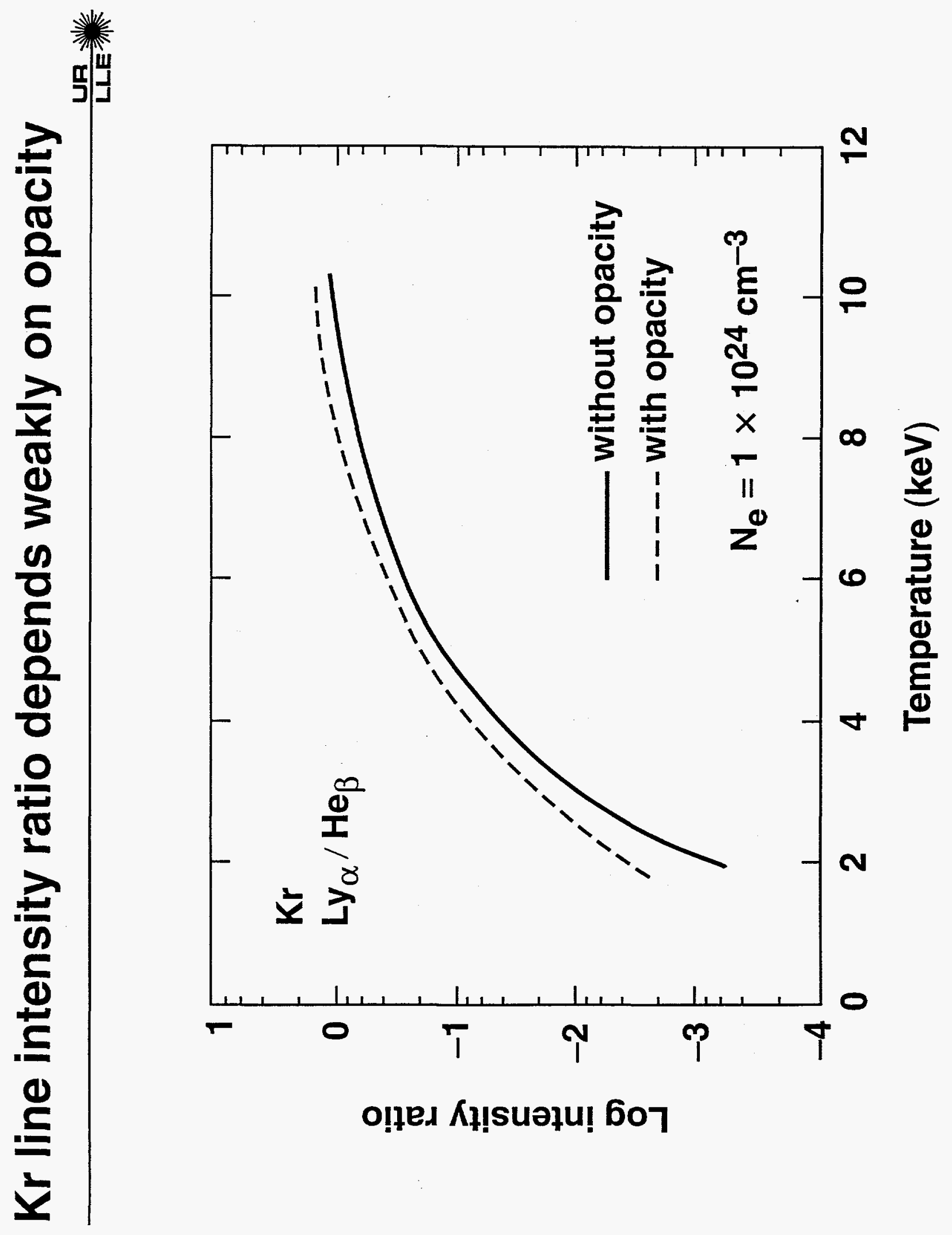

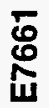

Fig. 8 


\section{DISCLAIMER}

This report was prepared as an account of work sponsored by an agency of the United States Government. Neither the United States Government nor any agency thereof, nor any of their employees, makes any warranty, express or implied, or assumes any legal liability or responsibility for the accuracy, completeness, or usefulness of any information, apparatus, product, or process disclosed, or represents that its use would not infringe privately owned rights. Reference herein to any specific commercial product, process, or service by trade name, trademark, manufacturer, or otherwise does not necessarily constitute or imply its endorsement, recommendation, or favoring by the United States Government or any agency thereof. The views and opinions of authors expressed herein do not necessarily state or reflect those of the United States Government or any agency thereof. 